\title{
PERFORMANCE OF XPERT MTB/RIF ASSAY FOR DETECTION OF M.TB IN PULMONARY AND EXTRA-PULMONARY SAMPLES IN INDIAN PATIENTS
}

\author{
Shetye S, Chheda P, Lad A, Matkar S \\ Metropolis Healthcare Ltd, Kurla West, Kirol Road, Mumbai, India
}

\begin{abstract}
Introduction: Conventional methods like Ziehl-Neelsen (ZN) staining and liquid culture have been the mainstay for diagnosis of Tuberculosis (TB). The gold standard Liquid Culture method has a longer turnaround time. In the wake of the TB catastrophe, newer rapid and easily accessible methods of detection are the need of the hour. A molecular method like the Xpert MTB/RIF assay has revolutionized the early and rapid diagnosis of TB. Objective of the current study was to assess the performance and utility of Xpert MTB/RIF assay for the diagnosis of $M$. tuberculosis in pulmonary and extra-pulmonary clinical specimens in a large Indian reference laboratory.

Methodology: The reference methods used were MGIT Liquid Culture system and ZN smear microscopy. Our study was performed in Global Reference Laboratory, Metropolis Healthcare, Mumbai, India for a period of 18 months with consecutive one thousand and forty two (518 Pulmonary + 524 extra-pulmonaryspecimens) clinical specimens obtained from the patients with clinical suspicion of tuberculosis. Diagnostic performance (sensitivity, specificity, positive predictive value (PPV) and negative predictive value (NPV) of the three methods were calculated with standard formulae.
\end{abstract}

Results: In comparison to MGIT Liquid culture, sensitivity of Xpert MTB/RIF assay for pulmonary and extra pulmonary specimens were $87.18 \%$ and $68.92 \%$, respectively while in comparison to ZN smear microscopy the sensitivity of Xpert MTB/RIF assay for pulmonary and extra pulmonary specimens were $92.67 \%$ and $83.81 \%$, respectively.

Conclusion: Our study concludes that in combination with the MGIT culture, Xpert MTB/RIF assay will significantly improve the detection rate of MTB bacteria.

Key words: Mycobacterium tuberculosis; Pulmonary; Extra-pulmonary; Xpert MTB/RIF assay, ZN staining

\section{INTRODUCTION}

Tuberculosis (TB) is a global public-health issue aggravated by the emergence of drug resistance strains. $^{1}$ After Acquired Immuno deficiency Syndrome (AIDS), TB is the greatest killer of human population due to a single infectious agent M. Tuberculosis. ${ }^{2}$ Morbidity and Mortality from TB have been counted the highest from Low- and

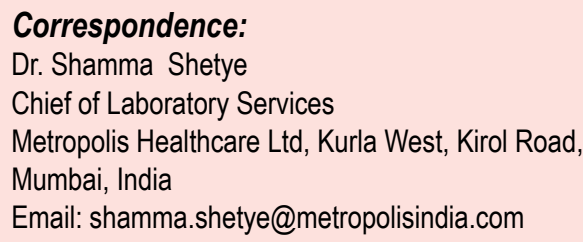

Middle-income countries. The African Region had $28 \%$ of the world's cases in 2014 , but the most severe burden relative to population: 281 cases for every 100,000 people, more than double the global average of $133 .{ }^{3}$

India is under the world's largest burden of tuberculosis (TB), accounting for one-fourth (24\%) of the global TB incidence. The global annual incidence estimate is 8.8 million cases, of which 1.5 million cases are from India. ${ }^{4}$ Another issue faced by developing countries like India, is the emergence of drug resistant tuberculosis (DR-TB) particularly multidrug resistant TB (MDR-TB) and extensively drug resistant TB (XDR-TB). The disease usually affects the lungs (pulmonary TB). Extra-pulmonary TB is defined as TB affecting other sites of the body. 
Recommended treatment for drug-susceptible TB is the combined use of first-line drugs; isoniazid, rifampicin, ethambutol and pyrazinamide for a period of six-months. ${ }^{1}$ Isoniazid and rifampicin are the powerful anti-TB drugs. TB bacteria resistant to isoniazid and rifampicin are indicative of multidrug resistant tuberculosis (MDR-TB). Treatment for MDR-TB is comparatively longer and includes second line anti-TB drugs. Rifampicin resistance is a surrogate marker for MDR- TB. ${ }^{5}$

Early rapid diagnosis of tuberculosis and appropriate use of recommended therapy is essential to control the emergence and spread of MDR and/or XDR strains. Conventional Acid-Fast Bacilli (AFB) smear microscopy by Ziehl-Neelsen (ZN) staining is a cost-effective method for the rapid identification of highly communicable TB patients. However, it is reported to be less sensitive as compared to the culture method. ${ }^{6}$ It gives no information about antibiotic resistance, viability and identification of the bacilli. Large MTB bacilli loads $(105 / \mathrm{ml})$ are required for a positive smear result. Culture medium is considered as the "gold standard" for TB diagnosis. ${ }^{7}$ It is more sensitive method than smear microscopy, detecting lower mycobacterial loads, for species identification. Accurate detection of TB bacilli and drug susceptibility can also be performed by culture method. ${ }^{8}$ However, longer time taken for detection of TB bacilli and species identification is its major constraint for routine TB diagnosis.

A molecular method, the Xpert $₫$ MTB/RIF assay (Cepheid, USA), was endorsed in 2010 by the World Health Organization (WHO), for the rapid identification of $M$. tuberculosis and antibiotic resistance in clinical specimens. ${ }^{9}$ The Xpert MTB/ RIF is a rapid molecular biologygene based assay that fully automates sample processing, DNA amplification and detection. It performs a real-timePCR reaction within a single closed cartridge. It requires very shorthands-on time (<2hours) and can be performed by operators with minimum technical expertise. Revised National Tuberculosis Control Program (RNTCP) is a state-run tuberculosis control, an initiative of the Government of India. Post-completion of feasibility study, RNTCP has endorsed the policy of prioritizing to offer rapid molecular test Xpert MTB/RIF (CBNNAT) to all presumptive TB cases for early diagnosis of TB as well as Rifampicin resistance. Introduction of Xpert MTB/RIF assay has revolutionized the diagnosis of tuberculosis (TB) by simultaneously detecting the bacteria and resistance to rifampicin (rif), a marker for multi-drug resistant TB (MDR-TB) as well as one of the principal first-line anti-tuberculosis drugs.

The aim of this study was to determine the sensitivity and specificity of the Xpert MTB/RIF assay for the diagnosis of MTB in pulmonary and extra-pulmonary clinical specimens registered at Global Reference Laboratory, Metropolis Healthcare, Mumbai, India. The results obtained by the Xpert MTB/RIF assay and ZN smear were compared with the results obtained by MGIT liquid culture.

\section{METHODOLOGY}

\section{Study population and samples:}

This study was carried out at Global Reference Laboratory, Metropolis Healthcare, Mumbai, India for a period of 18 months from June-2014 through December-2015. In our study, consecutive one thousand and forty two (1042) clinical specimens obtained from the patients with clinical suspicion of tuberculosiswere included. The number of male (557) and female (485) subjects were included in the study. Age (years) range of male was 9 to 91 years, whereas that of female was 01 month to 87 years. Average age of the subjects in the study was 39.52 years.

\section{Inclusion criteria:}

- Patients with clinical suspicion of tuberculosis

- Patient should be able to give at least their clinical history

- At least, the specimen material should be $3 \mathrm{ml}$ for expectorated sputum sample

- At least, $3 \mathrm{ml}$ sample volume for any kind of body fluids

- At least of $1 \mathrm{~cm} \times 1 \mathrm{~cm}$ of tissue specimen

\section{Exclusion criteria:}

- Patients provided insufficient sample volume.

- Samples received without request of all three tests.

\section{Follow up samples were not included in this study.}

The collected clinical specimens (1042) comprised of pulmonary (518) and extrapulmonary (524) specimens. The types of extra 
pulmonary specimens included Pus, abscess and aspirates, Body fluids (pleural fluids, ascitic fluids, cerebrospinal fluid, synovial fluid, urine and gastric fluids), Endometrium tissue, Lymph Node tissue, Spinal tissue and Bone Biopsy. The minimum volumes of sample required were as follows: $3 \mathrm{ml}$ for expectorated sputum sample, $3 \mathrm{ml}$ for any kind of body fluids and $1 \mathrm{~cm} \times 1 \mathrm{~cm}$ of tissue specimen. The sample was divided into 3 parts. Two parts were assigned to 2 different technologists, one in the Mycobacteriology laboratory, where the technologist read smears and inoculated cultures, and the other part in the molecular research laboratory, where the technologist performed the Xpert MTB/RIF assay, thus blinding the technologists to the results of other tests. The third part was stored at $2^{\circ} \mathrm{C}$ to $8^{\circ} \mathrm{C}$ for rechecking and reanalysis, if required.

\section{ZN smear and MGIT culture:}

The samples were processed fordigestion and decontamination using $4 \% \mathrm{~N}$-acetyl-L-cysteine and sodium hydroxide (NALC-NAOH) by the modified Petroff method ${ }^{10}$ and centrifuged. The speed of centrifuge was $3800 \mathrm{rpm}$ and time of centrifuge was 15 mins, after decontamination. After centrifugation, the sediment was re-suspended in 1.0 to $1.5 \mathrm{ml}$ of sterile phosphate buffer ( $\mathrm{pH} 6.8)$. The Mycobacteria Growth Indicator Tube (MGIT) manual mentioned an addition of 1.0 to $2.0 \mathrm{ml}$ as re-suspending volume of phosphate buffer. This suspension was used for inoculation and cultivation in liquid medium culture system MGIT [mycobacteria growth indicator tube] Bactec 960 culture; BD Microbiology Systems, USA. The volume of re-suspended sample used for MGIT was $0.5 \mathrm{ml}$ and the volume used for LJ culture was $0.1 \mathrm{ml}$. The tubes were incubated in the MGIT 960 instrument at $37^{\circ} \mathrm{C}$. The specimens were also analysed by light microscopy after ZiehINeelsen (ZN) staining of smear to investigate the presence of acid fast bacilli. The smear was checked for acid-fast bacilli under oil immersion and reported according to the Revised National Tuberculosis Control Program (RNTCP laboratory module). ${ }^{11}$ Solid culture was used only as backup and results were not separately analysed.

\section{Xpert MTB/RIF assay:}

The Xpert MTB/RIF assay was performed as described by Boehme et al. ${ }^{12}$ Sample reagent (SR) buffer was added (2:1 ratio) to all the unprocessed test specimens in $15 \mathrm{ml}$ falcon tube and the tube was manually agitated twice during a 15 minute incubation period at room temperature. Then, $2 \mathrm{ml}$ of the reaction material was transferred to the test cartridge by a sterile disposable pipette (provided with kits). Cartridges were loaded in the GeneXpert device and the automatically generated results were obtained after $114 \mathrm{~min}$. The interpretation of data from Xpert MTB/RIF assay was software based and not user dependent. The result was obtained in a simple text format which could be read easily. In cases where results were reported as being invalid, no result or error, the sample was reprocessed and rerun when sufficient material was available. Samples with insufficient material for reprocessing were not included in the study. For this study, samples were sufficient for rerun in all such cases.

\section{Statistical analysis:}

Diagnostic performance (sensitivity, specificity, positive predictive value (PPV) and negative predictive value (NPV) of the three methods were calculated with standard formulae. When results were indeterminate and a sufficient amount of the sample remained, the assay was repeated once, and the second result was used for analysis. A negative result for smear microscopy was reported if no acid-fast bacilli were detected in at least 200 observation fields. Cultures in MGIT liquid media were judged to have a negative result if no mycobacterial growth was seen until 6 weeks after incubation. A tuberculosis negative result for the Xpert MTB/RIF assay was generated automatically following an interpretative algorithm with the Gene Xpert Dx software. Apart from calculating binary (positive, negative) variables for all the test outcomes, the results were processed to analyse them quantitatively.

\section{RESULTS}

Our study evaluated the diagnostic accuracy of Xpert MTB/RIF assay both for pulmonary and Extra-pulmonary TB cases and compared it with the conventional techniques. Out of the 1042 samples analysed in our study, 454 samples $(43.57 \%)$ were Xpert positive which included 341 (75.11\%) ZN smear positive and $113(24.88 \%)$ Zn smear negative cases. Whereas, culture positive cases accounted for 451 (43.28\%) including 305 (67.62\%) ZN smear positive and 146 (32.37\%) Zn smear negative entries (Table 1). 


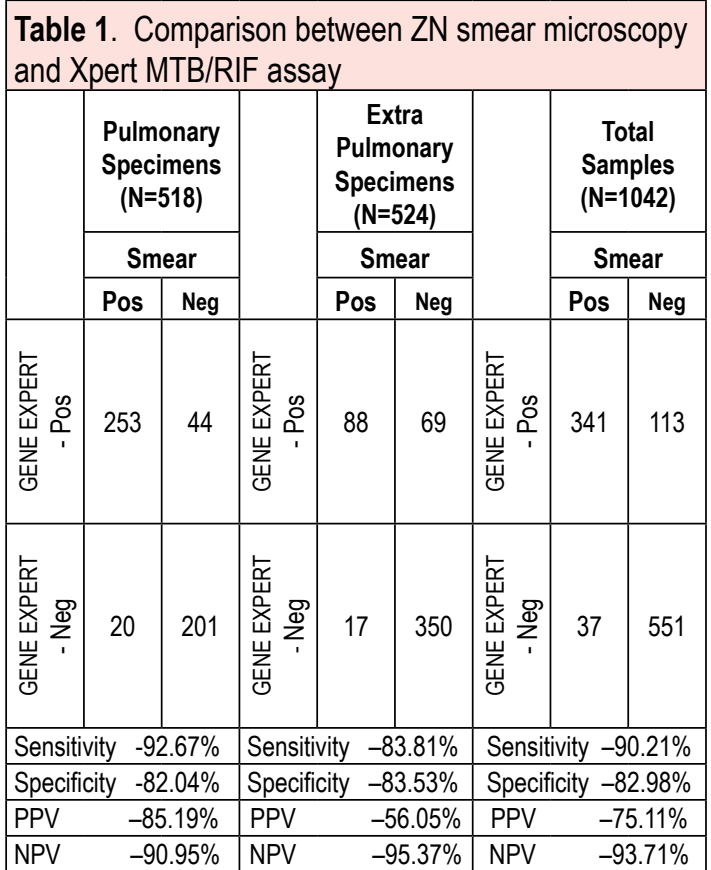

Our findings on the performance of the Xpert MTB/ RIF assay corroborated well with studies regarding accurate detection of MTBC bacilli in AFB-negative specimens. ${ }^{17}$

Our study recorded 294 (28.21\%) cases (235 pulmonary and 59 extra-pulmonary) which were smear positive, culture positive and Xpert MTB/RIF assay positive. This observation is in accordance with the studies carried out by Boehme et al $(2011)^{18}$, Boehme et al $(2010)^{12}$ and Helb D et al (2010). ${ }^{19}$

Using liquid culture as the gold standard for comparison, the overall sensitivity of Xpert MTB/ RIF assay was $79.74 \%$ (87.18\% for pulmonary specimens and $68.92 \%$ for extra-pulmonary specimens). The specificity of Xpert MTB/RIF assay was recorded at $86.25 \%$ (Table 2).

The results on the performance of Xpert MTB/RIF assay correlated well with previous studies ${ }^{20,21,22}$ and ${ }^{23}$ that report the sensitivity and specificity for pulmonary specimens ranging from $67 \%$ to 90 , and $94 \%$ to $100 \%$ respectively. The heterogeneity between studies may reflect differences between the patient population, patient selection, type of pulmonary and extra-pulmonary specimens and the quality of the samples. ${ }^{24}$

Our study also reported thirty one specimens that were smear positive but remained negative by Xpert MTB/RIF assay. Out of the 31 smear positive and Xpert MTB/RIF assay negative specimens, 19

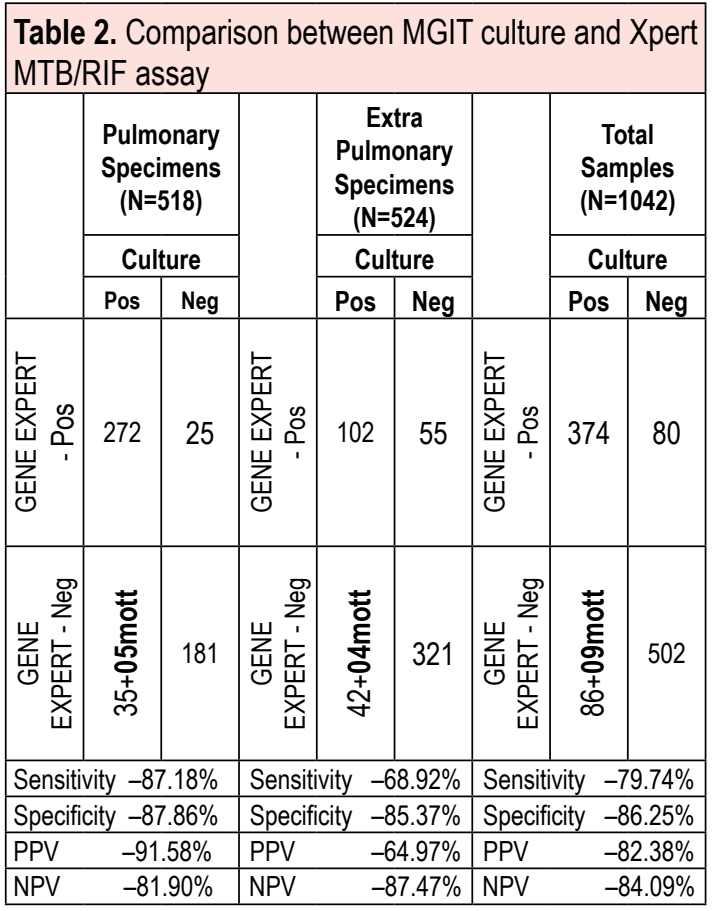

of them were also negative for MTB culture. On the contrary, one hundred and thirteen specimens were not detected by smear, but were identified by Xpert MTB/RIF assay. Out of the 113 such cases, 80 cases were also detected positive by MGIT liquid culture while 33 cases remain undetected by MGIT liquid culture. This indicates superior sensitivity of Xpert MTB/RIF assay over ZN smears. Similar observations have been reported in studies by Scott et al (2011). ${ }^{25}$

Thirteen samples which remain undetected by both ZN smear microscopy and MGIT liquid culture were detected by Xpert MTB/RIF assay. Out of these 13 cases, 11 were extra-pulmonary and 02 were pulmonary samples. Thus, Xpert MTB/RIF assay detects cases which remain undetected by ZN smear and culture. In our study, only twelve specimens including pulmonary (05) and extrapulmonary (07) were positive on culture and smear but negative on Xpert MTB/RIF assay. The reason for false negative Xpert MTB/RIF assay results may be due to the limited number of bacilli in those particular samples. Out of 12 cases, 03 cases grew Mycobacteria other than culture (MOTT) other than MTB complex in culture. The other 09 cases were smear positive but paucibacillary (scanty grading) and hence this could be the reason for negative Gene-xpert result. Also, it is possible that stressed bacilli in patients may grow in culture. 


\section{DISCUSSION}

One of the most critical steps in tuberculosis management is the rapid and accurate laboratory diagnosis of mycobacterium tubercle (MTB) complex. It helps to achieve the most appropriate treatment strategy for TB. World Health Organization (WHO) recommended Xpert MTB/ RIF assay as a point-of-care testing for TB bacilli. Xpert MTB/RIF assay is rapid assay which provides diagnostic result for TB and RIF resistance within two hours. It is less prone to contamination; requires minimal biosafety facilities; can be performed by technicians with little training and no risk of laboratory cross-contamination because of its closed-cartridge design. ${ }^{13}$ Studies report a high sensitivity in smear-negative pulmonary TB which is particularly relevant for patients with HIV infection. The objective of our study was to determine the sensitivity and specificity of the Xpert MTB/RIF assay for the diagnosis of MTB in pulmonary and extra-pulmonary clinical specimens in comparison to MGIT liquid culture system and ZN smear microscopy. Published literature has suggested high sensitivity and specificity for Xpert MTB/RIF assay, primarily in smear positive specimens..$^{14,15,16}$

The International Standards for Tuberculosis Care (ISTC) recommends that patients suspected of having pulmonary TB should submit at least two sputum specimens for bacteriological examination. One of these samples should be obtained early morning, because the sample would have the highest yield at that time.$^{26}$ One of the reasons for low sensitivity of $\mathrm{ZN}$ smear microscopy is due to the fact that $105 / \mathrm{ml}$ is required for AFB to be seen using smear microscopy. However it has been reported that multiple sputum tests can give a sensitivity of about $90 \% .^{27}$

The XpertMTB/RIF assay is assumed to be specific; it only detects DNA from intact $M$. tuberculosis bacilli and contamination from free DNA is thought to be removed by a washing step. The assay, however, does not differentiate between viable, dormant, and non-viable intact $M$. tuberculosis bacilli that are shed during effective anti-tuberculosis treatment. ${ }^{28}$ In our findings-high rates of Xpert MTB/RIF assay positive results suggest that even DNA fragments from lysed or damaged bacteria could have been detected by Xpert MTB/RIF assay. This observation suggested that the Xpert MTB/RIF assay could be a complimentary test to culture for the diagnosis of TB. The paucibacillary nature of extra-pulmonary specimens with a tendency of $M$. tuberculosis to form clumps leads to an uneven distribution of the bacilli during cultures. Also, there is loss of viable bacilli during NALC-NaOH processing (due to decanting supernatant steps), unlike Xpert processing, wherein the entire volume of the processed specimen is used; and the Xpert sample reagent has a better homogenization and liquefaction efficiency than NALC-NaOH processing. ${ }^{28}$ Xpert MTB/RIF assay is a method with high specificity and false positive results may be explained by the detection of dead MTB that would not be detected on culture or the fact that this method has better sensitivity.

Xpert MTB/RIF assay detects both live and dead bacteria. ${ }^{29}$ False positive results may be explained by the detection of dead MTB that would not be detected on culture. Cross-contamination is known to be one of the reasons for false positive results. However, PCR in Xpert MTB/RIF is less prone to contamination due to the closed reaction chamber. ${ }^{15}$ Furthermore, the surfaces where the specimens are processed were extensively cleaned to avoid contamination with bacterial DNA. For reliable results a good quality of specimen collection is very important. A patient with a negative Xpert MTB/RIF result can still have TB.

The diagnostic accuracy of the Xpert MTB/RIF assay in the extra-pulmonary specimens couldn't be analysed by specimen's origin due to lack of adequate number of specimens. Also, definitive diagnosis becomes difficult due to paucibacillary nature of TB bacilli in extra-pulmonary specimens. ${ }^{30}$

In the present study, the combined sensitivity of Xpert MTB/RIF assay was $88.14 \%$. A systematic review and meta-analyses conducted by Denkingeret al ${ }^{31}$ showed that Xpert MTB/RIF assay has a sensitivity ranging from $50 \%$ to $100 \%$ with pooled sensitivity of $83 \%$. More recently, Penzet al ${ }^{32}$ reviewed 36 studies in their meta-analyses and confirmed Xpert MTB/RIF assay pooled sensitivity of $87 \%$ that is similar to our study. ${ }^{25}$ However, the sensitivity of Xpert MTB/RIF assay in the current study is lower than what was found in similar study by Ligthelmet al (sensitivity, 96.7\%). ${ }^{33}$ 


\section{Limitations}

Our study was conducted retrospectively and hence the results could not be correlated with radiological findings and histo-pathological reports. The sensitivity and specificity of MTB/RIF assay to detect Rifampicin resistance in our study could not be evaluated in our study as Rifampicin sensitivity by phenotypic method was not performed for all the positive samples.

\section{CONCLUSION}

Conventional methods like ZN smear microscopy and culture are laborious and require more time to establish clinical diagnosis of tuberculosis. Though the sensitivity of microscopy can be increased, still a large fraction remains undetected for TB. Several efforts are carried out to expand the coverage area of Xpert MTB/RIF assay usage across the world. Grants and subsidies are being provided by Government's and various organizations to enable the availability of Xpert MTB/Rif assay in developing and underdeveloped nations where healthcare systems face economic constraints. Our study concluded that the Xpert MTB/RIF assay displays better sensitivity than $\mathrm{ZN}$ smears for early detection of MTB infection. However, the Xpert MTB/RIF assay results must always be confirmed by MGIT liquid culture. In combination with the MGIT culture, Xpert MTB/Rif assay will definitely improve the detection rate of MTB bacteria. This will ensure an overall increase in the detection rate and better sensitivity for diagnosis of MTB.

Conflict of Interest: We, the authors declare no conflicts of interest.

\section{REFERENCES}

1. Borrell Sand Gagneux S. Strain diversity, epistasis and the evolution of drug resistance in Mycobacterium tuberculosis. ClinMicrobiol Infect 2011;17:815-20.

2. Ducati $R$ G, Ruffino-Netto $A$, Basso $L A$, et al. The resumption of consumption - a review on tuberculosis.MemInstOswaldo Cruz 2006;101:697714.

3. World Health Organization. Global Tuberculosis Report, 20 $2{ }^{\text {th }}$ edition, 2015.

4. World Health Organization: Global Tuberculosis Control: Epidemiology, Strategy and Financing. WHO report, 2010.
5. Raoot A and Dev G. Evaluate "Rifampicin Resistance" as Surrogate Marker for Rapid Detection of MDR-TB Using Real-Time PCR Directly on FNAC Samples of Tuberculous Lymphadenitis. British Journal of Medicine \& Medical Research 2015;9:1-8.

6. Singhal R, Myneedu VP. Microscopy as a diagnostic tool in pulmonary tuberculosis. International Journal of Mycobacteriology 2015;4:1-6

7. Joint Committee for the Revision of Korean Guidelines for Tuberculosis, Korea Centers for Disease Control and Prevention. Korean guidelines for tuberculosis. 2nd ed. Seoul and Cheongwon: Joint Committee for the Revision of Korean Guidelines for Tuberculosis, Korea Centres for Disease Control and Prevention; 2014.

8. European Centre for Disease Prevention and Control. Mastering the basics of TB Control: Development of a Handbook on TB diagnostic methods. Stockholm: ECDC; 2011.

9. Lawn SD, Nicol MP. Xpert@ MTB/RIF assay: development, evaluation and implementation of a new rapid molecular diagnostic for tuberculosis and rifampicin resistance. Future Microbiology $2011 ; 6: 1067-1082$.

10. Cruickshank $R$, Duguid IP, Maimion BP, Swain RHA. Edinburgh: Churchill Livingstone; 1975. Medical Microbiology. $12^{\text {th }}$ edition.

11. RNTCP central TB division, Manual for Laboratory technicians, New Delhi, India: Directorate General of Health Services, Ministry of Health and Family Welfare, 1998.

12. Boehme C, Nabeta P, Hillemann D, et al. Rapid Molecular Detection of Tuberculosis and Rifampin Resistance. N Engl J Med 2010; 363:1005-15.

13. Weyer K, Mirzayev F, Migliori GB, et al. Rapid molecular TB diagnosis: evidence, policy making and global implementation of Xpert MTB/RIF. EurRespir J 2013:42:252-71.

14. Ozkutuk N, Surucüoglu S. Evaluation of the Xpert MTB/RIF assay for the diagnosis of pulmonary and extrapulmonary tuberculosis in an intermediateprevalence setting. MikrobiyolBul 2014;48:223232.

15. Hillemann D, Rüsch-Gerdes $S$, Boehme $C$, et al. Rapid molecular detection of extra-pulmonary tuberculosis by the automated Gene Xpert MTB/ RIF system. J ClinMicrobiol 2011;49:1202-05.

16. Shagufta I, Asyia Z, Hussain S. Rapid diagnosis of tuberculosis using Xpert MTB/RIF assay - Report from a developing country. Pak J Med Sci 2015; 31:105-110. 
17. Moure R, Muñoz L, Torres M, et al. Rapid Detection of Mycobacterium tuberculosis complex and rifampin resistance in smear-negative clinical samples by use of an integrated real-time PCR method. J ClinMicrobiol 2011;49:1137-39.

18. Vadwai V, Boehme $C$, Nabeta $P_{1}$ et al. Xpert MTB/ RIF: a new pillar in diagnosis of extra-pulmonary tuberculosis? J Clin Microbiol 2011;49:2540-45.

19. Helb $D$, Jones $M$, Story $E$, et al. Rapid detection of Mycobacterium tuberculosis and Rifampin Resistance by use of On-Demand, Near-Patient Technology. J ClinMicrobiol 2010;48:229-37.

20. Ioannidis $P$, Papaventsis D, Karabela $S$, et al. Cepheid GeneXpert MTB/RIF Assay for Mycobacterium tuberculosis detection and rifampin resistance identification in patients with substantial clinical indications of tuberculosis and smear-negative microscopy results. J ClinMicrobiol 2011;49:3068-70.

21. Al-Ateah SM, Al-Dowaidi MM and El-Khizzi NA.. Evaluation of direct detection of Mycobacterium tuberculosis complex in respiratory and nonrespiratory clinical specimens using the Cepheid Gene Xpert ${ }^{\circledR}$ system. Saudi Med J 2012; 33:110005 .

22. Walusimbi S, Bwanga F, De Costa A, et al. Metaanalysis to compare the accuracy of GeneXpert, MODS and the WHO 2007 algorithm for diagnosis of smear-negative pulmonary tuberculosis. BMC Infect Dis 2013; 13:507. doi: 10.1186/1471-233413-507

23. Laura MS, Larke N, Petrs JA, et al. Diagnostic accuracy of the Xpert MTB/RIF assay for extrapulmonary and pulmonary tuberculosis when testing non-respiratory samples: a systematic review. BMC Infectious Diseases 2014;14:709.

24. Lawn S D, Zumla Al. Diagnosis of extra-pulmonary tuberculosis using the Xpert ${ }^{\circledR}$ MTB/RIF assay. Expert Rev Anti Infect Ther 2012;10:631-35.

25. Lesley E S, McCarthy K, Gous N, et al. Comparison of Xpert MTB/RIF with Other Nucleic Acid Technologies for Diagnosing
Pulmonary Tuberculosis in a High HIV Prevalence Setting: A Prospective Study. PLoS Medicine 2011;8:e1001061. doi: 10.1371/journal. pmed.1001061

26. Hopewell P C, Fair E L, Uplekar M. Updating the International Standards for Tuberculosis Care Entering the Era of Molecular Diagnostics. Ann Am ThoracSoc 2014;11:277-85.

27. Tuberculosis Control as an Integral part of primary health care. World Health Organization, Geneva. 1988. ISBN 9241542446.

28. Cheng V. C, Yam Y C, Hung I F N, et al. Clinical evaluation of the polymerase chain reaction for the rapid diagnosis of tuberculosis. J. Clin. Pathol 2004;57:281-85.

29. Miotto P, Bigoni S, Migliori GB, et al. Early tuberculosis treatment monitoring by $\operatorname{Xpert}(\mathrm{R})$ MTB/RIF. EurRespir J 2012;39:1269-71.

30. Purohit M, Mustafa T. Laboratory Diagnosis of Extra-pulmonary Tuberculosis (EPTB) in Resourceconstrained Setting: State of the Art, Challenges and the Need. J ClinDiagn Res 2015;9:EE01EE06.

31. Denkinger CM, Schumacher SG, Boehme CC, et al. Xpert MTB/RIF assay for the diagnosis of extrapulmonary tuberculosis: a systematic review and meta-analysis. European Respiratory Journal 2014;44:435-46.

32. Penz E, Boffa J, Roberts D, et al. Diagnostic accuracy of the Xpert@ MTB/RIF assay for extrapulmonary tuberculosis: A meta-analysis. The International Journal of Tuberculosis and Lung Disease 2015;19:278-84.

33. Ligthelm LJ, Nicol MP, Hoek KG, et al. Xpert MTB/ RIF for rapid diagnosis of tuberculous lymphadenitis from fine-needle-aspiration biopsy specimens. Journal of Clinical Microbiology 2011;49:3967-70. 